\title{
Case Series Study on the Use of BU Pharmacopuncture Treatment in Patients with Acute Lumbar Sprain
}

\author{
Muhack Yang ${ }^{1}$, Jongwon Jang ${ }^{1}$, Eunhye Cha', Byungsoo Ahn'ㄹ, Younghee Bang ${ }^{3}$, \\ Beomyong Song ${ }^{4}$, Jiyong Shin ${ }^{5}$, Chankeun Ahn ${ }^{6}$, Deokho Kim ${ }^{7}$, Sungchul Kim ${ }^{1 *}$
}

\author{
${ }^{1}$ PDepartment of Acupuncture \& Moxibustion Medicine, Wonkwang University Gwangju Korean Medicine Hospital, Gwangju, Korea \\ ${ }^{2}$ Anjung Korean Medicine Clinic, Seoul, Korea \\ ${ }^{3}$ Sol Korean Medicine Clinic, Jinju, Korea \\ ${ }^{4}$ Department of Acupuncture \& Moxibustion Medicine, Woosuk University Korean Medicine Hospital, Jeonju, Korea \\ ${ }^{5}$ Doil Korean Medicine Clinic, Seoul, Korea \\ ${ }^{6}$ Yeongdeung Korean Medicine Hospital, Iksan, Korea \\ ${ }^{7}$ Department of Korean Medicine, Graduate School of Wonkwang University, Iksan, Korea
}

\section{Key Words}

SAcute Lumbar Sprain, BU Pharmacopuncture(BUP),

Oswestry Disablity Index(ODI), Visual Analogue Scale(VAS)

\begin{abstract}
Objective: The purpose of this study was to investigate the clinical effects of BU pharmacopuncture therapy consisting of bear's gall(fel ursi) and ox bezoar(bovis calculus) on acute lumbar sprain.
\end{abstract}

Methods: 12 patients diagnosed as acute lumbar sprain in 6 designated local Korean medicine clinics from October 2017 to February 2018 were treated by BU pharmacopuncture. Several acupoints in abdomen and lumbar region were selected by clinicians at their own discretion. The effectiveness of the therapy was evaluated using VAS and ODI. After that we reviewed the medical records of all these patients to evaluate the effectiveness and safety of the therapy.

Results: The VAS and ODI scales were significantly decreased after BU pharmacopuncture therapy. And no major complications and adverse effects were reported.

Received: Dec 23, 2017 Reviewed: May 2, 2018 Accepted: May 6, 2018

(c) This is an Open-Access article distributed under the terms of the Creative Common Attribution Non-Commercial License (http://creativecommons.org/licenses/by-nc/4.0/) which permits unrestricted noncommercial use, distribution, and reproduction in any medium, provided the original work is properly cited.

( This paper meets the requirements of KS X ISO 9706, ISO 9706-1994 and ANSI/NISO Z39.48-1992 (Permanence of Paper).
Conclusion: BU pharmacopuncture showed rapid pain relief in patients with acute lumbar sprain. It is possible to shorten the treatment period of acute lumbar sprain and prevent progressing to chronic back pain in advance. To establish the effects of BU pharmacopuncture therapy, more succeeding clinical and laboratory studies are needed.

\section{Introduction}

Low back pain became major health problem in modern society, and caused huge medical expenses, loss of labor force. Acute low back pain usually resolves on its own and patients can get better and return to work within one month, and constant improvements occur for following three months. But, if symptoms don't subside during this period, pain and disability would be prolonged and most patients suffer recurrence of pain within 12 months [1].

Pharmacopuncture is a form of therapy consisted of two traditional therapeutic methods, herbal medicine and acupuncture therapy [2]. Lee's paper announced that BU pharmacopuncture(BUP) therapy tends to activate the autonomic nervous system and control sympathetic nerve system within normal range [3]. Shin's 
article illuminated the effectiveness of Sweet Bee Venom pharmacopuncture treatment combined with acupuncture on sprain of lumbar spine in comparison to single acupuncture treatment [4]. Seo's paper mentioned BUP therapy combined with cupping has effect on low back pain [5]. Kim's study showed the effectiveness of Bee Venom pharmacopuncture therapy on lumbar sprain [6].

This study was designed to investigate the effects of the sole Bovis Calculus-Fel Ursi(BU) pharmacopuncture therapy for acute lumbar sprain. 12 patients diagnosed with ALS were selected at 6 local Korean medicine clinics.

\section{Case series report}

\subsection{Materials and methods 2.1.1. Study subject}

BAmong outpatients visiting 6 designated local Korean medicine clinics from October 2017 to February 2018, we examined patients diagnosed with acute lumbar sprain. Written consent forms that patients agree to use of their medical record for this study were received. Patients only who met the following inclusion criteria were included. Patients met any of the following exclusion criteria were excluded.

\subsubsection{Inclusion criteria}

1 Subject whose chief complaint is acute lumbago developed in the last 3 days

2 Diagnosed as acute lumbar sprain in medical clinics

3 Diagnosed as acute lumbar sprain, but took no other treatments before their visit

4 Does not have allergic reaction to skin test

5 Agrees to participate into this study

\subsubsection{Exclusion criteria}

(1) Suffered for more than 3 days

(2) Took any treatment before their visit

(3) Has abnormal findings in his/her MRI/X-ray/CT image

(4) Has chief complaints other than lumbago

(5) Has past history of the following: HIVD, spinal stenosis, spondylolysis, etc.

(6) Has a history of schizophrenia

(7) Has a history of cancer

(8) Positive reaction to any of SLRT, Patrick's test, Piriformis test

(9) Otherwise excluded by the decision of researcher.

\subsubsection{Study method}

\subsubsection{BUP therapy}

Total 2ml BUP was injected into several acupoints in each patient at practitioner's discretion. Any treatment other than BUP was not applied. The content of Ursodeoxycholic Acid(UDCA), the marker compound of Fel Ursi, was measured using Ultra performance liquid chromatography-UV (Fig. 1). The concentration of UDCA in BUP is estimated as $3.47 \mathrm{mg} / \mathrm{mL}$.

\subsubsection{Evaluation of effect \\ 2.1.3.1. VAS}

The severity of pain was evaluated by $100 \mathrm{~mm}$ Visual Analogue Scale(VAS). Patients marked scale between 0 and $100 \mathrm{~mm}$ on their own.

\subsubsection{Oswestry disability index(ODI) KOR Ver.}

ODI is a multiple choice questionnaire felt in by a patient and consists of 10 questions related to each activity in daily life. Each question is scored on a scale of $0-5$ with the statements describing the disability of daily life

\subsubsection{Statistics process}

The results of study were statistically processed using $\mathrm{R}$ version 3.4.3(2017-11-30). Shapiro- Wilk's test was used for normality test of VAS and ODI. As a result, these two variables did not disprove null hypothesis $(p>0.05)$ and proved to be under normality. The pre-treatment VAS, ODI values and endpoint VAS, ODI values were compared using paired two sample t- test.

\subsection{Results}

\subsubsection{Demographic characteristics}

Of a total of 12 subjects, there were 7 male and 5 female enrolled in this study. The mean age of the patient was $45.8 \pm 10.1$ years. The mean BMI of the patients was $23.3 \pm 3.1\left(\mathrm{~kg} / \mathrm{m}^{2}\right)$. Other demographic, social, and clinical characteristics of the study patients was also investigated (Table 1).

\subsubsection{Treatment result}

\subsubsection{Selected acupoints}

After every treatment session, practitioners made records of acupoints they chose. The most selected acupoint was ST25, and this acupoint was used by every 6 practitioners. Generally, ST25, BL23, BL24, BL25 were the most selected 4 acupoints for treatment. And 6.16 acupoints were used every session of treatment by one practitioner on average (Fig. 2).

\subsubsection{Safety evaluation}

To evaluate the safety of BUP, adverse effect was checked at every visit of subject. There were no major complications, and no adverse effect was reported during our study.

\subsubsection{Average number of visits and treat- ment period}


The average number of treatment session was $2.4 \pm 1.0$. The mean treatment period from the first visit to the last visit was 3.1 \pm 1.7 days (Table 2 ).

\subsubsection{VAS, ODI score change}

The mean VAS score in patients before treatment was $7.8 \pm 2.3$ and after the last treatment, $1.7 \pm 1.5$. The mean amount of VAS score change was -6.1 . The mean ODI score in patients before treatment was $20.2 \pm 9.6$ and after the last treatment, 5.3 \pm 5.7 . The mean amount of ODI score change was -14.9 (Table 3 ). These results showed a significant decrease after BUP treatment (Fig. 3).

\section{Discussion}

Pharmacopuncture is a new acupuncture treatment in traditional Korean medicine (TKM) combining acupuncture based on the meridian theories and herbal medicine based on Qi and flavor theories [7]. Pharmacopuncture injects herbal medicine extract at acupuncture points related to diseases, tender points, or positive reaction points based on meridian theory. Pharmacopuncture treats diseases by regulating body function and improving the pathological condition of the body [8]. The clinical trials and studies of pharmacopuncture therapy have made great progress in Korea. Research on the types of pharmacopuncture and which diseases can be dealt with pharmacopuncture has to be extended [9].

Lumbar region has wide range of motion and constant mechanical stress is applied to this region. Lumbar lordosis gradually shifts into sacral kyphosis and these two region consists lumbosacral joint. Thus, the large amount of movement in lumbar region is compromised by the small amount of movement in sacral region, and these properties in lumbosacral joint make lumbosacral region unstable, vulnerable to injury [10].

We examined the 12 patients diagnosed as acute lumbar strain or sprain among outpatients visiting 6 designated local Korean medicine clinics from October, 2017 to February, 2018. We applied BUP therapy to them and evaluated the effectiveness of the therapy by the scale of VAS shift and ODI change. Safety of the therapy is assessed with the frequency of occurrences of adverse effect.

In this study, the mean VAS score in patients before treatment was $7.8 \pm 2.3$ and after the last treatment, $1.7 \pm 1.5$. The mean amount of VAS score change was -6.1 . The mean ODI score in patients before treatment was 20.2 \pm 9.6 and after the last treatment, 5.3 \pm 5.7 . The mean amount of ODI score change was 14.9. This result shows BUP is effective in treating acute lumbar sprain or strain and there is no significant adverse effect of it.

This study has the limitations of small sample size, lack of control group and lack of objective evaluation indicators except for VAS, ODI. To establish the effectiveness of BUP, more succeeding studies with a large amount of samples in the form of randomized controlled trials are needed.

\section{Acknowledgements}

This study was supported and funded by National Devel- opment Institute of Korean Medicine (NIKOM)

\section{Conflict of interest}

The authors declare that there are no conflicts of interest 
Table 1 Demographic characteristics

\begin{tabular}{lc}
\hline Characteristics & Value \\
\hline Total N & 12 \\
Sex & \\
Male & $7(58.3 \%)$ \\
Female & $5(41.7 \%)$ \\
Age (years) & $45.8 \pm 10.1$ \\
Height (cm) & $166.6 \pm 8.0$ \\
Weight (kg) & $65.3 \pm 13.4$ \\
BMI (kg/m $\left.{ }^{2}\right)$ & $23.3 \pm 3.1$ \\
Tobacco use & \\
None & \\
Smoker & \\
Alcohol Use & $5(58.3 \%)$ \\
None & $5(41.7 \%)$ \\
\hline
\end{tabular}

Table 2 Average number of visits and treatment period

\begin{tabular}{lc}
\hline & Value (days) \\
\hline The number of visits & $2.3 \pm 1.0$ \\
\hline Treatment period & $3.1 \pm 1.7$ \\
\hline The values are means \pm SD &
\end{tabular}

Table 3 VAS, ODI score change

\begin{tabular}{lll}
\hline & \multicolumn{1}{l}{ VAS } & ODI \\
\hline Visit 1 & $7.8 \pm 2.3$ & $20.2 \pm 9.6$ \\
Endpoint & $1.7 \pm 1.5$ & $5.3 \pm 5.7$ \\
Change & -6.1 & -14.9 \\
t-statistic & -9.61 & -5.17 \\
& $1.10 \mathrm{E}-06$ & $3.11 \mathrm{E}-04$ \\
p-value & $(\mathrm{p}<0.001)$ & $(\mathrm{p}<0.001)$ \\
\hline & \\
\hline The values are means $\pm \mathrm{SD}$
\end{tabular}

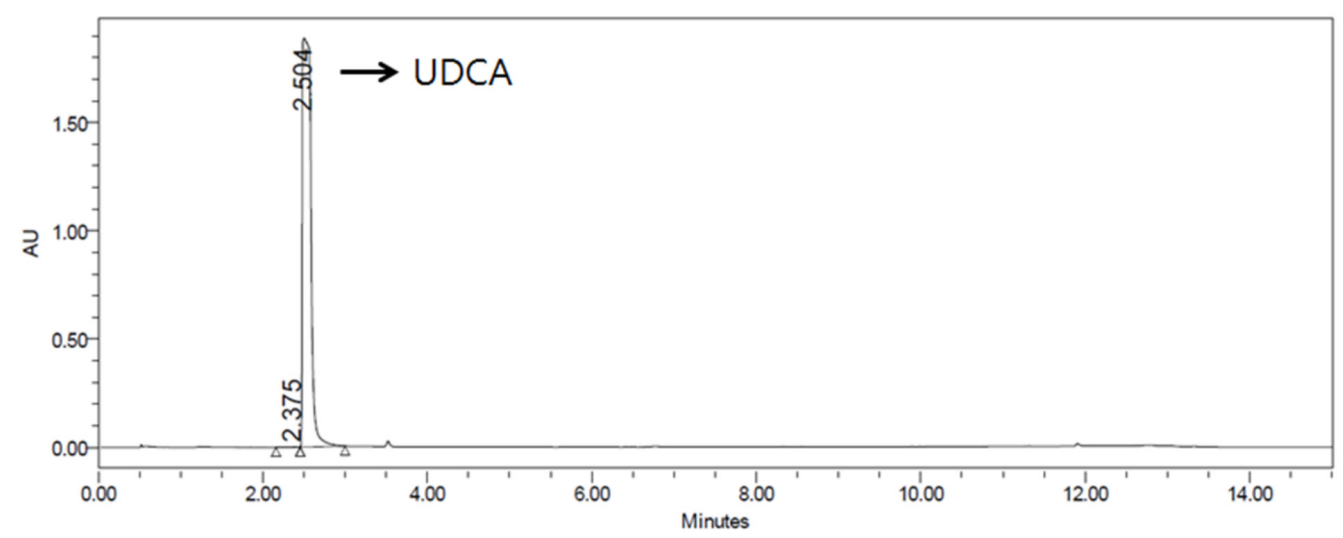

Figure 1 UDCA component analysis in BU extracts using Ultra performance liquid chromatography-UV 


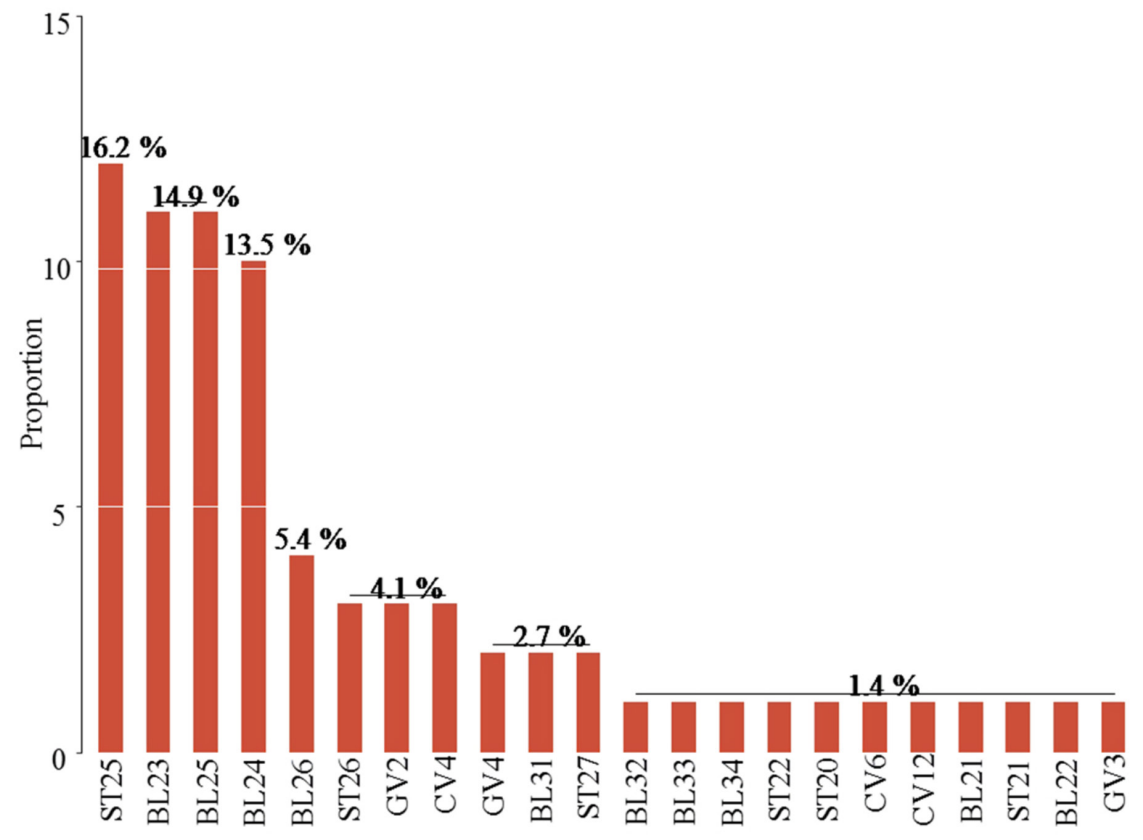

Figure 2 The frequency and proportion of used acupoints for treatment
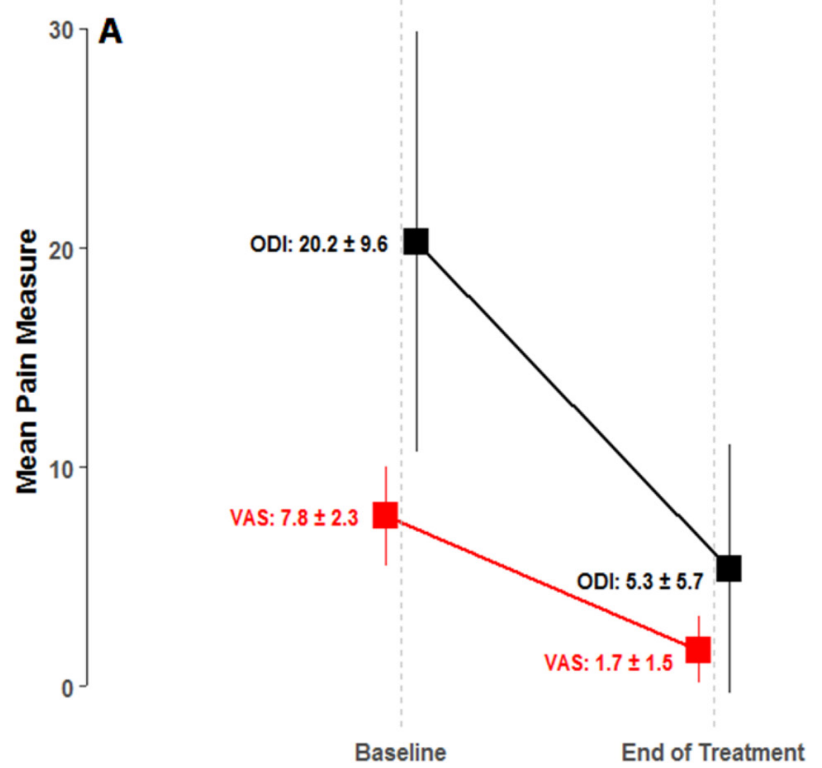

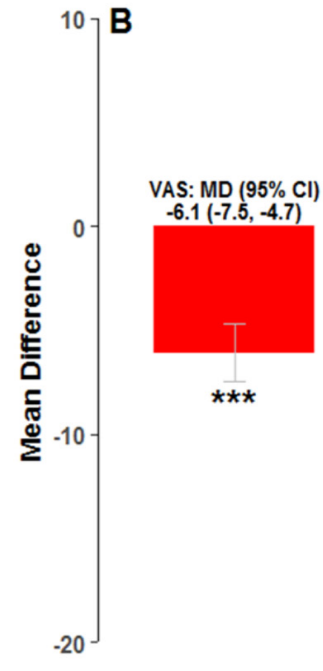

VAS

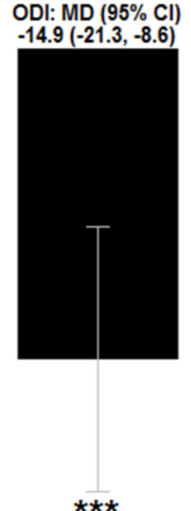

ODI

Figure 3 A) Estimated mean (squares) and SD (lines on squares) of VAS and ODI at the baseline and end of treatment. B) Mean difference between baseline and end of treatment for VAS and ODI (bars) and their $95 \%$ confidence intervals (gray error bars). The asterisks below the error bars indicate the magnitude of $p$-values, which were derived from paired two sample t-test. ${ }^{* * *}: \mathrm{p}<0.001$

Abbreviation: MD, mean difference; CI; confidence interval 


\section{References}

1. Andrea D. Furlan, Maurits van Tulder, Dan Cherkin, Hiroshi Tsukayama, Lixing Lao, Brian Berman, Acupuncture and Dry-Needling for Low Back Pain: An Updated Systematic Review Within the Framework of the Cochrane Collaboration. Spine. 2005; 30(8):944-63

2. Kim JD, Kang DI. A Descriptive Statistical Approach to the Korean Pharmacopuncture Therapy. Journal of Acupuncture and Meridian Studies. 2010; 3(3):141-9

3. Lee HY, Lee JB, Cho YH, Song BY, Yook TH. The Effects of Cervi Pantotrichum Cornu Pharmacopuncture and Bovis calculus $\nabla F e l$ Ursi Pharmacopuncture on the Heart Rate Variability. The Journal of Korean Acupuncture \& Moxibustion Society. 2010; 27(1):65-74

4. Shin YJ. A Clinical Pilot Study Comparing Sweet Bee Venom parallel treatment with only Acupuncture Treatment in patient diagnosed with lumbar spine sprain. Journal of Pharmacopuncture. 2011; 14(2):37-43

5. Seo JC, Seo BM, Lee YK, Choi SH, Kim KW, Yoon JS, et al. The Effects of BU Herbal Acupuncture on Low Back Pain of Sprain. Journal of Pharmacopuncture. 2005; 8(2):5-10

6. Kim KT, Song HS. The Effectiveness of Bee Venom Acupuncture Therapy on the Treatment of Sprain of L-spine. The Journal of Korean Acupuncture \& Moxibustion Society. 2005; 22(4):113-120

7. Korean pharmacopuncture institute. Pharmacopuncturology: principles and clinical applications. Seoul: Elsevier Korea LLC; 2012. p. 3-5. Korean.

8. Lee $\mathrm{MH}$, Son IC. Introduction to the aqua-acupuncture therapy and problems. The Acupuncture. 1998;15(2):511-518

9. Lee KH, Cho YY, Kim SC, Sun SH. History of Research on Pharmacopuncture in Korea. Journal of Pharmacopuncture. 2016;19(2): 101-108

10. Yoon DY, Son JM, Choi JS, Jung SH, Kim SJ. Effect of Chuna Treatment(Manipulation) on Lumbar Sprain Caused by Traffic Accident. The Journal of Korea CHUNA Manual Medicine for Spine and Nerves. 2010; 5(1): 27-8 